\title{
ON A REALIZATION OF PRIME TANGLES AND KNOTS
}

\author{
QUACH THI CAM VAN
}

The notion of a prime tangle is introduced by Kirby and Lickorish [7]. It is related deeply to the notion of a prime knot by the following result in [8]: summing together two prime tangles gives always a prime knot.

The purpose of this paper is to exploit this above mentioned result of Lickorish in creating or detecting prime knots which satisfy certain properties. First, we shall express certain knots (two-bridge knots and Terasaka slice knots [14]) as a sum of a prime tangle and an untangle (the existence of such a sum is proven to every knot in [7] and is not unique) in a natural way (natural means here depending on certain specific geometrical characters of the class of knots). Second, every Alexander polynomial (or Conway polynomial) is shown to be realized by a prime algebraic knot (algebraic in the sense of Conway [3], BonahonSiebenmann [2]) which can be expressed as the sum of two prime algebraic tangles. Finally, an application of these prime tangles and knots can be found in realizing Alexander polynomial concordance by a concordance of prime knots, first shown by Bleiler [1].

\section{Definitions and notations.}

(a) Tangles. The notion of tangle is introduced by Conway [3].

Definition. A (two-string) tangle is a pair $(B, t)$ where $B$ is a 3 -ball and $t$ is a pair of disjoint spanning arcs in $B(t \cap \partial B=\partial t)$. A tangle $(B, t)$ is untangled if the two arcs of $t$ are unknotted and if there is a properly embedded disc in $B(D \cap \partial B=\partial D)$ which separates the two arcs of $t$.

Every untangle can be constructed in the following way: starting from the corner points of a square "pillowcase", which are on the boundary of a 3-ball $B$, draw $p / q$ slope lines on its front and $-p / q$ slope lines on its back for a certain $p / q \in Q \cup 1 / 0$ (Figure 1); we shall denote it by $T_{p / q}=\left(B, t_{p / q}\right)$. The single or two-component link, produced by joining outside the diagram the top right of $t_{p / q}$ to its bottom right and the top left to its bottom left, is called the link $K_{p / q}$ associated to the tangle $T_{p / q}$ (Figure 1). Every two-bridge knot is a link $K_{p / q}$ associated to a tangle $T_{p / q}$ with $p$ even and $q$ odd ([11, p. 114], [12], [13]).

Received January 26, 1982 and in revised form August 20, 1982. This work was supported by the Swiss National Fund for Scientific Research. 

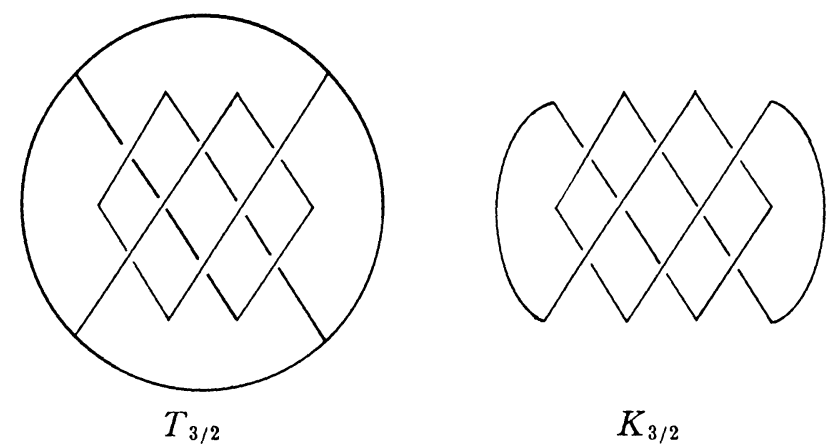

FIGURE 1

Given two tangles $\left(B_{1}, t_{1}\right)$ and $\left(B_{2}, t_{2}\right)$, a new tangle can be produced by identifying a (disc, point pair of $\partial t_{1}$ ) in the boundary of $B_{1}$ to a (disc, point pair of $\left.\partial t_{2}\right)$ in the boundary of $B_{2}$. The result is called a partial sum of the tangles $\left(B_{1}, t_{1}\right)$ and $\left(B_{2}, t_{2}\right)$. If one makes the choice of identification as depicted in Figure 2a, one obtains the Conway sum of tangles $T=(B, t)$ and $T^{\prime}=\left(B^{\prime}, t^{\prime}\right)$. It is denoted by $T+T^{\prime}$.

Given two tangles $\left(B_{1}, t_{1}\right)$ and $\left(B_{2}, t_{2}\right)$ let $f:\left(\partial B_{1}, \partial t_{1}\right) \rightarrow\left(\partial B_{2}, \partial t_{2}\right)$ be a homeomorphism. A single or two-component link can be created by identifying the boundaries of the tangles via $f$. The result $\left(B_{1}, t_{1}\right) \cup$, $\left(B_{2}, t_{2}\right)$ is called a sum of tangles. If one makes the choice of identification illustrated in Fig. 2b (which consists practically to join the tangles by bands), the resulting link is called, in Bleiler's terminology [1], the join of tangles $\left(B_{1}, t_{1}\right)$ and $\left(B_{2}, t_{2}\right)$.

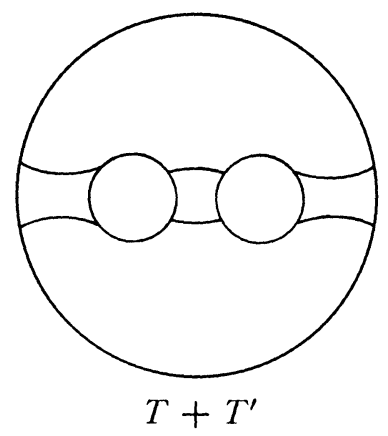

(a)

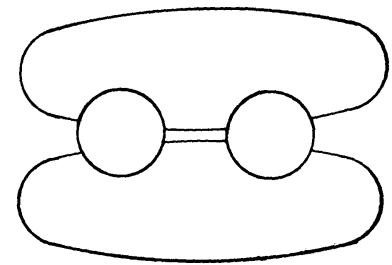

(b)

Figure 2

If a tangle $T=(B, t)$ carries the string orientation shown in Figure 3a (the entry and exit points alternate), the two ways to complete $T$ in an oriented knot or link depicted in Figure $3 \mathrm{~b}$ and in Figure 3c, are called respectively the denominator $D_{T}$ and numerator $N_{T}$ of $T$. 


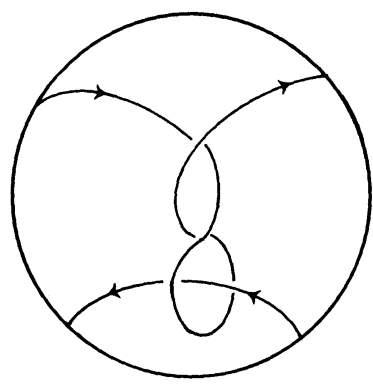

(a)

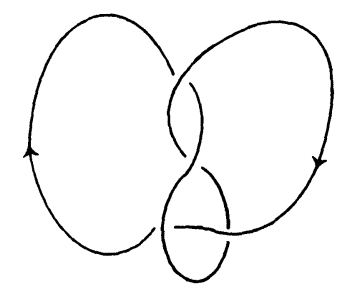

(b)

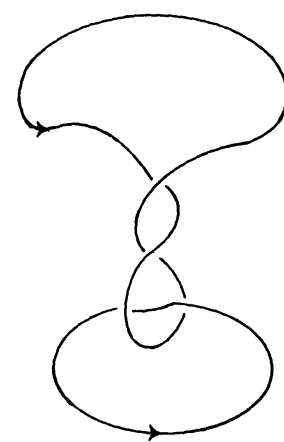

(c)

FigURE 3

In [7], the definition of a prime tangle is given as follows:

Definition. A tangle $(B, t)$ is prime if it satisfies the following properties: Any 2 -sphere in $B$, which meets $t$ transversely in two points, bounds in $B$ a ball meeting $t$ in an unknotted $\operatorname{arc} ;(\alpha)(B, t)$ is not untangled.

(b) Algebraic tangles and knots (algebraic in the sense of [3], [2]):

A band is an embedding of $S^{1} \times[0,1]$ or the Moëbius band in $S^{3}$ such that its core is unknotted.

Definition. Let $A_{1}$ and $A_{2}$ be two bands and $F$ a 2 -sphere separating $S^{3}$ in 3-balls $B_{1}$ and $B_{2}$ such that $A_{1} \subset B_{1}, A_{2} \subset B_{2}$ and $\partial B_{1} \cap A_{1}=$ $\partial B_{2} \cap A_{2}$ is a square on which the intersection of the bands' cores is transverse. The surface $A_{1} \cup A_{2}$ is a plumbing of bands $A_{1}$ and $A_{2}$ (it is a special case of Murasugi plumbing [10]). If $S$ is an (non-)orientable surface obtained by bands plumbing, a weighted graph $G$ can be associated such that:

the vertices of $G$ represent the bands and their weights the number of half-twists of the corresponding bands;

two vertices of $G$ are connected by an edge if and only if there is a plumbing of the corresponding bands.

$G$ is called a plumbing graph.

Definition. A knot is algebraic if it is the boundary of a surface obtained by bands plumbing according to a plumbing graph which is a tree. Equivalently, a knot is algebraic if it results from a sum of an untangle with a tangle obtained by partially summing a finite collection of untangles together. A tangle is algebraic if it is obtained by partially summing a finite collection of untangles.

2. (a) Let $T_{p_{1} / q_{1}}$ and $T_{p_{2} / q_{2}}$ be two untangles. Consider the Conway $\operatorname{sum} T_{p_{1} / q_{1}}+T_{p_{2} / a_{2}}$. 
Lemma 1. If $p_{1} / q_{1}$ and $p_{2} / q_{2} \notin \mathbf{Z} \cup 1 / 0$ and if $q_{1}$ and $q_{2}$ are odd, $T_{p_{1} / q_{1}}$ $+T_{p_{2} / q_{2}}$ is a prime tangle.

Remark. If $q_{1}$ or $q_{2}=1$, for instance $q_{2}$, the Conway sum $T_{p_{1} / q_{1}}+T_{p_{2} / q_{2}}$ results in the untangle $T_{\left(p_{1}+p_{2} q_{1}\right) / q_{1}}$. If there is just one $p_{i} / q_{i}=1 / 0$, for instance $p_{2} / q_{2}$, the tangle $T_{p_{1 / q_{1}}}+T_{1 / 0}$ is neither prime nor untangled. If $p_{1} / q_{1}=p_{2} / q_{2}=1 / 0$ the Conway sum $T_{1 / 0}+T_{1 / 0}$ is not a two-string tangle.

Proof of Lemma 1. An untangle can be added to $T_{p_{1} / q_{1}}+T_{p_{2} / p_{2}}$ to produce the connected sum of knots $K_{p_{1} / q_{1}}$ and $K_{p_{2} / q_{2}}$. Thus the Conway sum $T_{p_{1} / q_{1}}+T_{p_{2} / q_{2}}$ is not untangled.

The join of tangles $T_{p_{1} / q_{1}}$ and $T_{p_{2} / q_{2}}$ gives a two-bridge knot or link $K_{p / q}$ which is the boundary of a surface obtained by bands plumbing according to the following linear tree:

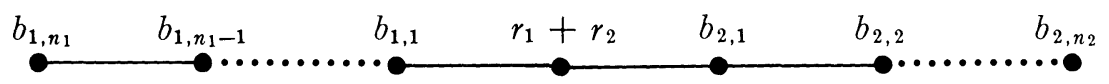

where $b_{i, m}$ and $r_{i}$ appear as integer coefficients of a continued fraction expansion of $p_{i} / q_{i}=r_{i}+\left[b_{i, 1} \ldots b_{i, n_{i}}\right]$ with

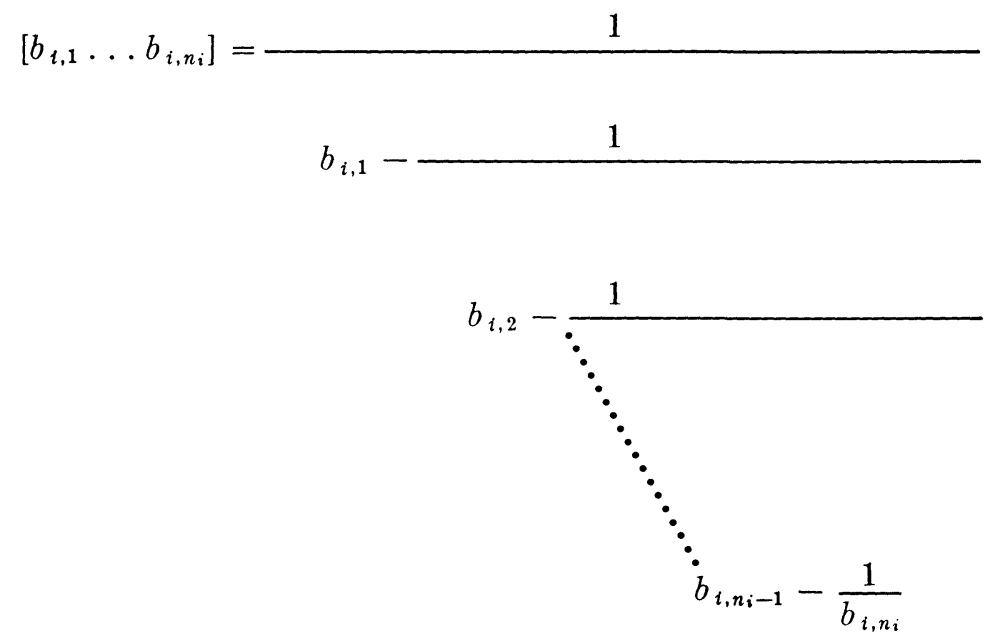

If $p_{1}$ and $p_{2}$ are of the same parity, $q=p_{1} q_{2}+p_{2} q_{1}$ is even, therefore $K_{p / q}$ is a link (see for example [13]). Since a two-bridge link has both of its components unknotted, the existence of a knotted arc-ball pair in the tangle $T_{p_{1} / q_{1}}+T_{p_{2} / q_{2}}$ could not be possible. Hence $T_{p_{1} / q_{1}}+T_{p_{2} / q_{2}}$ is, in this case, a prime tangle.

If $p_{1}$ and $p_{2}$ are not of the same parity, suppose (without loss of generality) that $p_{1}$ is even and $p_{2}$ odd. Consider now the Conway sum

$$
T_{p_{1} / q_{1}}+T_{p_{2} / q_{2}}+T_{1 / 1}
$$


which is, as already remarked, equivalent to the sum

$$
T_{p_{1} / q_{1}}+T_{p_{2}{ }^{\prime} / q_{2}} \text { with } p_{2}^{\prime}=p_{2}+q_{2} .
$$

$p_{1}$ and $p_{2}{ }^{\prime}$ are now of the same parity, then, by the discussion given above, $T_{p_{1} / q_{1}}+T_{p_{2} / q_{2}}$ is prime. Finally, a theorem of Lickorish [8, Theorem 3] implies that $T_{p_{1 / q 1}}+T_{p_{2}^{\prime} / q_{2}}$ is also a prime tangle.

Corollary 1. A two-bridge knot can be expressed as a sum of an untangle with a prime algebraic tangle.

Proof. Every two-bridge knot can be obtained as a knot $K_{p / q}$ associated to an untangle $T_{p / q}$ with $p$ even and $q$ odd such that

$$
p / q=\left[2 b_{1}, \ldots, 2 b_{n}\right], b_{i} \in \mathbf{Z}-0 \text { and } n \geqq 2 .
$$

A two-bridge knot $K_{p / q}$ can therefore be considered as the boundary of an orientable surface obtained by bands plumbing according to the tree

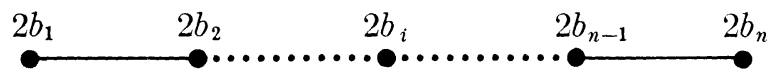

Let $p^{\prime} / q^{\prime}=\left[2 b_{1}-3,2 b_{2}, \ldots, 2 b_{n}\right]$ be obtained from $p / q$ by replacing the first coefficient $2 b_{1}$ by $2 b_{1}-3$. Consider now the Conway sum $T_{p^{\prime} / q}+T_{1 / 3}$. By Lemma 1, the Conway sum $T_{p^{\prime} / q^{\prime}}+T_{1 / 3}$ is a prime tangle which is, by construction, algebraic. It is easy to see that the join of $T_{p^{\prime} / q^{\prime}}$ and $T_{1 / 3}$ produces the two-bridge knot $K_{p / q}$.

Remarks. Consider now the partial sum of $T_{p^{\prime} / q^{\prime}}+T_{1 / 3}$ with the untangle $T_{1 / 1}$ as illustrated in Figure 4. The arcs of the resulting tangle admit an orientation which allows us to speak about the denominator and numerator of the considered tangle denoted by $S_{p / q}$. The numerator of $S_{p / q}$ restitutes also the knot $K_{p / q}$.

The tangle, Conway sum of two untangles described in Lemma 1, is in fact a particular case of algebraic tangles, the primeness of which can be shown by the passage to two-fold cyclic branched coverings $[10]$ and by using the results of [15] and [8]. The above given proof avoids this sophisticated passage.

(b) Lemma 2. Let $\left(B_{0}, t_{0}\right)$ be a tangle (with string orientation depicted in Figure 3a) such that its numerator is the unlink and its denominator is not the trivial knot. Then $\left(B_{0}, t_{0}\right)$ is a prime tangle.

Proof. As the numerator of $\left(B_{0}, t_{0}\right)$ is, by assumption, the unlink, the condition $(\alpha)$ to be prime is satisfied.

Suppose that $\left(B_{0}, t_{0}\right)$ is an untangle; say $T_{p_{0} / q_{0}}$. Since the denominator and numerator of an untangle $T_{p / q}$ are respectively the single or two component link $K_{p / q}$ and $K_{q / p}$, to be an unlink when completed in the numera- 


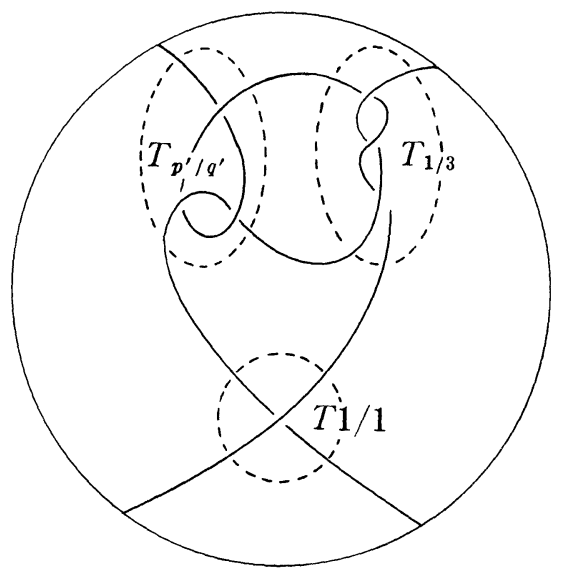

FIGURE 4

tor, the tangle $T_{p_{0} / q_{0}}$ must have $p_{0} / q_{0}=1 / 0$ and its denominator would be the trivial knot. Hence $\left(B_{0}, t_{0}\right)$ is not an untangle.

Let $A(t)$ be an Alexander polynomial which satisfies the Fox-Milnor condition [4]: there exists a polynomial $f(t) \in \mathbf{Z}\left[t, t^{-1}\right]$ such that

$$
A(t)= \pm t^{m} f(t) f\left(t^{-1}\right) .
$$

Corollary 2. Let $A(t)$ be an Alexander polynomial satisfying the FoxMilnor condition. Then there exists a tangle $G_{f}$ such that its numerator is the unlink and its denominator is a knot with Alexander polynomial

$$
A(t)= \pm t^{m} f(t) f\left(t^{-1}\right) .
$$

Proof. By Terasaka [14] there exists a slice knot $K_{f}$ constructed from two circles connecting by a band with Alexander polynomial

$$
A(t)= \pm t^{m} f(t) f\left(t^{-1}\right) .
$$

Let $C$ be a ball intersecting the connecting band as illustrated in Figure 5 . Hence $\left(C, C \cap K_{f}\right)$ is an untangle and, by construction, the tangle complement

$$
\left(S^{3} / C,\left(S^{3} / C\right) \cap K_{f}\right)=G_{f}
$$

has the required properties.

3. In the following, we shall use the Conway rather than the Alexander polynomial.

Recall that a knot Conway polynomial is

$$
\nabla(z)=1+\sum_{i=0}^{n} a_{i} z^{2 i} \text { with } a_{i} \in \mathbf{Z} .
$$




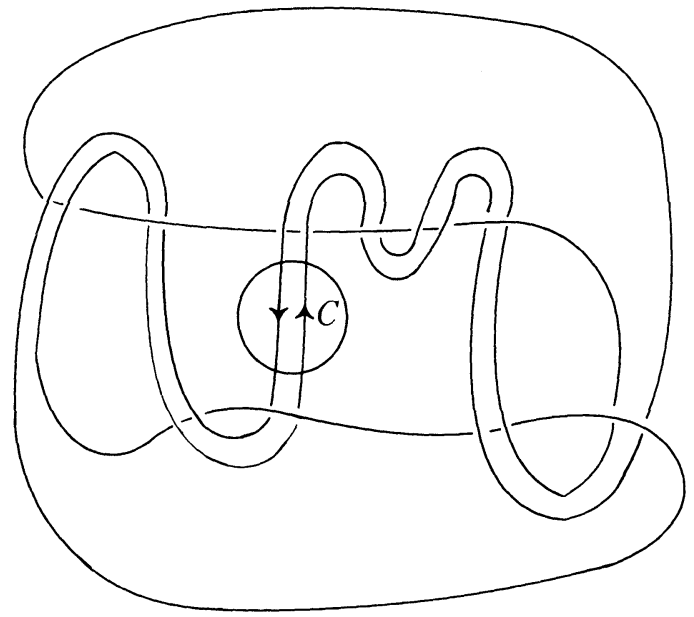

FIGURE 5

Proposition 3. Let

$$
\nabla(z)=1+(-1)^{l-1}\left\{\sum_{i=0}^{l-1}(-1)^{i} p_{i} z^{2(l-i)}\right\}
$$

with $p_{i} \in \mathbf{Z}$. Then there exists an algebraic knot with Conway polynomial $\nabla(z)$.

Proof. Consider the surfaces $F_{i}, i=0, \ldots, l$, depicted in Figure 6 with their respective plumbing graphs. The surface $S_{p_{0} \ldots p_{l-1}}$, illustrated in Figure 7 with its corresponding plumbing graph, is obtained by Murasugi plumbing of these surfaces $F_{i}, i=0, \ldots, l$. The knot $K_{p_{0}, \ldots, p}$, boundary of $S_{p_{0} \ldots p_{l-1}}$ is therefore, by construction, algebraic.

Exploiting Conway's methods of calculation (see [3] or [6]) and the property of $K_{p_{0}, \ldots, p_{l-1}}$ to be of unknotting number one (it suffices to replace the plumbing element $F_{0}$ of $S_{p_{0} \ldots p_{l-1}}$ by a non-twisted band), it is easy to prove by induction that the Conway polynomial of $K_{p_{0}, \ldots, p-1}$ is

$$
\nabla(z)=1+(-1)^{l-1}\left\{\sum_{i=0}^{l-1}(-1)^{i} p_{i} z^{2(l-i)}\right\} .
$$

Proposition 4. Let $K_{p_{0}, \ldots, p_{l-1}}$ be the knot constructed as above for Proposition 3, with $l \geqq 3$. Then:

(4a) there exists embedded in $S^{3}$ a 2-sphere cutting $K_{p_{0}, \ldots, p}$ in four points and bounding 3-balls $B_{1}$ and $B_{2}$ such that

$\left(^{*}\right) \quad\left(B_{i}, B_{i} \cap K_{p_{0}, \ldots, p_{l-1}}\right)$ is a prime tangle, $i=1,2$.

(4b) $K_{p_{0}, \ldots, p_{l-1}}$ is a prime knot.

Proof. By [8, Theorem 1], (4b) is merely a consequence of (4a). Therefore it suffices to prove $(4 a)$. 


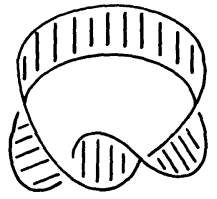

$F_{0}$

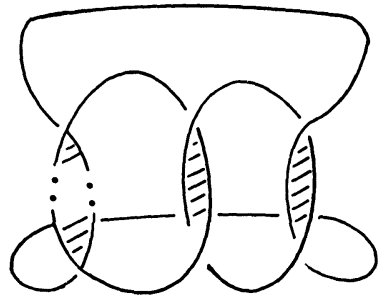

$F_{i}, i=1, \ldots, l-1$

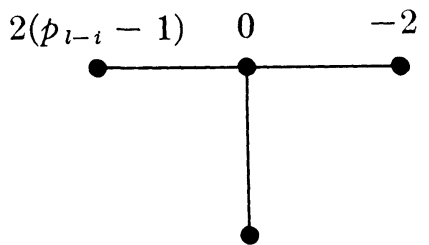

$+2$

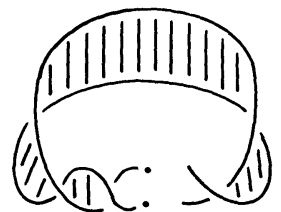

$F_{l}$

Figure 6

$\begin{array}{llllllll}+2 & 2\left(p_{l-1}-1\right) & -2 & 2\left(p_{i}-1\right) & -2 & 2\left(p_{1}-1\right) & -2 & 2\left(p_{0}-1\right)\end{array}$
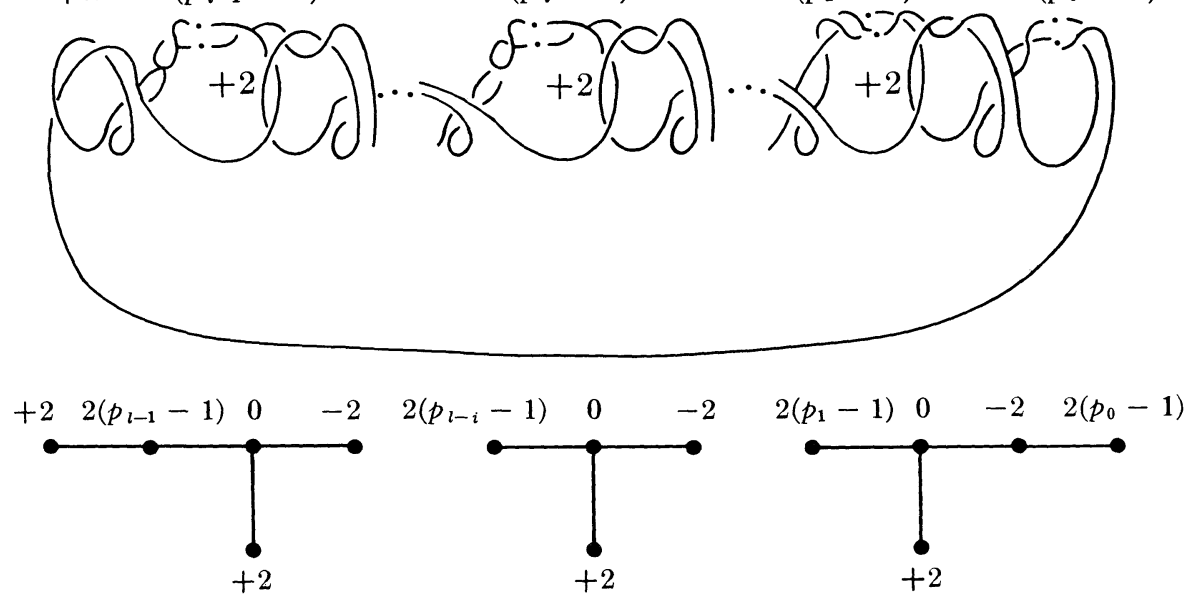

FiguRe 7

A regular projection of $K_{p_{0}, \ldots, p_{l-1}}$ is given by Figure 8 . Consider now the 2-sphere $F$ meeting $K_{p_{0}, \ldots, p_{l-1}}$ as depicted in Figure 8.

Assertion. $F$ is a 2 -sphere satisfying the property $(*)$.

By the knot's construction, the assertion can be proved by induction, directly in $S^{3}$ without passing to two-fold cyclic branched covering. 


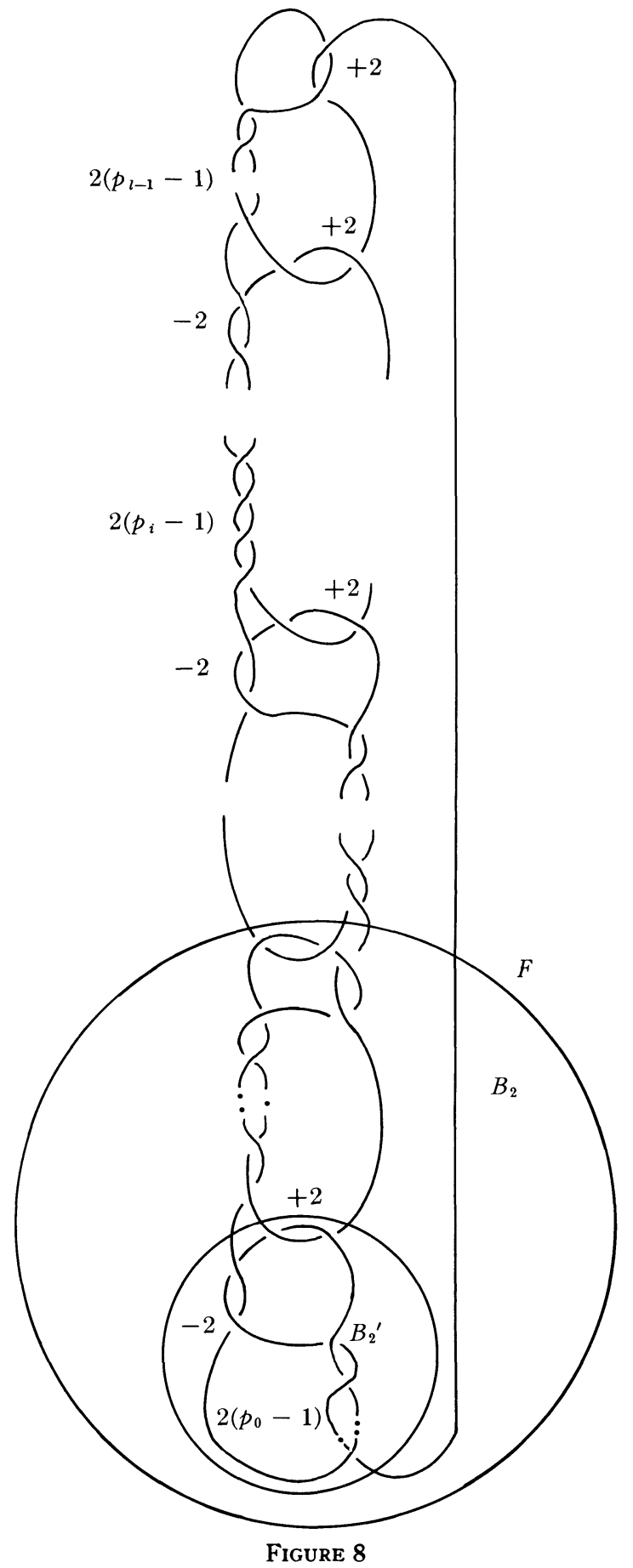


Consider the tangles $\left(B_{2}, B_{2} \cap K\right)$ with $\partial B_{2}=F$ and $\left(B_{2}{ }^{\prime}, B_{2}{ }^{\prime} \cap K\right)$ illustrated in Figure 8. $\left(B_{2}{ }^{\prime}, B_{2}{ }^{\prime} \cap K\right)$ is not an untangle, as its denominator produces a connected sum of links. Completing the tangle $\left(B_{2}{ }^{\prime}\right.$, $\left.B_{2}{ }^{\prime} \cap K\right)$ in numerator gives the trivial knot. Hence $\left(B_{2}{ }^{\prime}, B_{2}{ }^{\prime} \cap K\right)$ is prime. Therefore, by [8, Theorem 3], the tangle $\left(B_{2}, B_{2} \cap K\right)$ is also prime.

For $l=3$, it is easy to verify in the same way that the tangle complement

$$
\left(S^{3} \backslash B_{2}\left(S^{3} \backslash B_{2}\right) \cap K\right)
$$

is prime.

Suppose now that the assertion and therefore the proposition are proved until $l-1$ (until $K_{p_{0}, \ldots, p_{l-1}}$ ).

Denote the tangle complement to $\left(B_{2}, B_{2} \cap K_{p_{0}, \ldots, p_{l}}\right)$ by $U_{l}$. The denominator of $U_{l}$ gives the connected sum of $K_{p_{2}, \ldots, p_{l}}$ and a Hopf link. Hence $U_{l}$ is not untangled. The numerator $N\left(U_{l}\right)$ of $U_{l}$ is the knot $K_{p_{2}+1, p_{3}, \ldots, p_{l}}$. By assumption of induction, the knots $K_{p_{2}, \ldots, p_{l}}$ and $K_{p_{2}+1, \ldots, p_{l}}$ would be the same. Since the $p_{i}$ characterizing the two knots are different and therefore their Conway polynomials are, by Proposition 3 , not equal, it follows that such a knotted arc-ball pair could not exist.

Remark. The knots $K_{p_{0}, \ldots, p_{l-1}}$ are of unknotting number one. An affirmative answer to a problem in [5] would be an alternative proof for their primeness.

We are now in a position to prove the following theorem:

THEOREM 5. Let

$$
\nabla(z)=1+(-1)^{l-1}\left\{\sum_{i=0}^{l-1}(-1)^{i} p_{i} z^{2(l-i)}\right\}
$$

with $p_{i} \in \mathbf{Z}$. There exists a knot $K_{p_{0}, \ldots, p_{l-1}}$ with $\nabla(z)$ as Conway polynomial such that there exists embedded in $S^{3}$ a 2 -sphere satisfying the property $\left(^{*}\right)$.

Proof. For $l \geqq 3$, the theorem is given by Proposition 4 . For $l=1$ and 2 , consider the tangle $V$, depicted in Figure 9. It gives, when completed in denominator $D_{V}$, the connected sum of the pretzel knot $K(-3,5,7)$ with its image $r K(-3,5,7)$ where $r: S^{3} \rightarrow S^{3}$ is an orientation reversing homeomorphism. The Conway polynomials of the numerator $N_{V}$ and the denominator $D_{V}$ of $V$ are: $\nabla\left(N_{V}\right)=0$ and $\nabla\left(D_{V}\right)=1$.

Let $M_{2-l}, l=1,2$, be the following tangle: For $l=2$, it is the prime algebraic tangle $S_{p / q}$ described in a remark of Corollary 1 , the numerator of which is the knot $K_{p_{0}}$ of Proposition 3 (recall that $K_{p_{0}}$ is a two-bridge knot). For $l=1, M_{1}$ is the prime algebraic tangle depicted in Figure 10 which produces, when completed in numerator, the knot $K_{p_{0}, p_{1}}$ of Proposition 3 . 


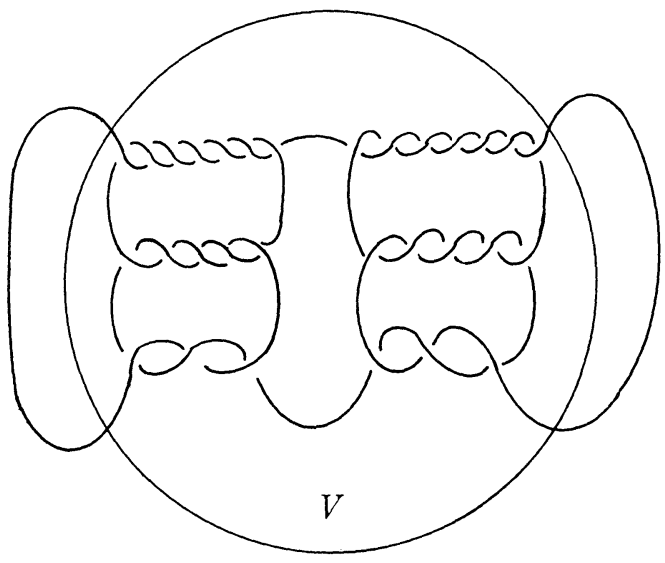

Figure 9

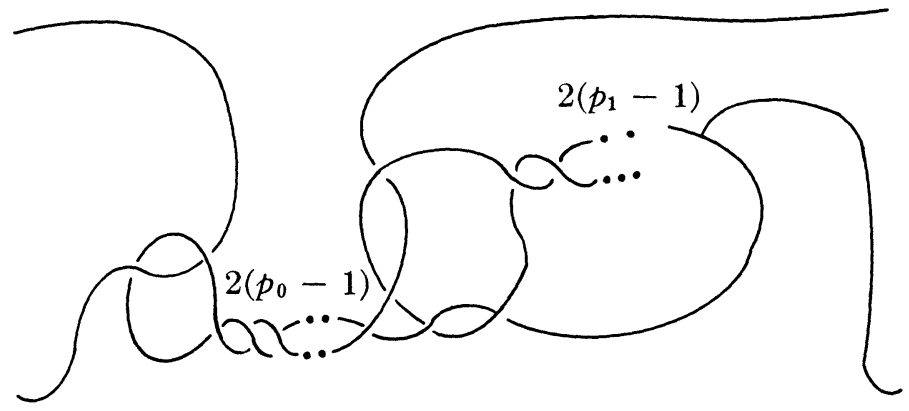

FigURE 10

Consider now the Conway sum of $V$ and $M_{i}, i=0,1$. The join of $V$ and $M_{i}$ (the numerator of $V+M_{i}$ ) (Figure 11) is the knot $K_{i}$ with Conway polynomial (see [3] or [6]):

$$
\begin{aligned}
\nabla\left(K_{i}\right)=\nabla\left(N_{V}\right) \nabla\left(D_{M_{i}}\right)+\nabla\left(N_{M_{i}}\right) & \nabla\left(D_{V}\right)=\nabla\left(N_{M_{i}}\right) \\
& = \begin{cases}1+p_{0} z^{2} & i=0, \\
1+p_{1} z^{2}-p_{0} z^{4} & i=1 .\end{cases}
\end{aligned}
$$

By construction, the knot is ribbon-concordant (in terminology of Gordon) to $K_{p_{0}}$ when $i=0$ and $K_{p_{0}, p_{1}}$ when $i=1$, algebraic and by [8, Theorem 1], prime.

Consider now the join of the tangles $G_{f}$ and $C_{p_{l}}$ where:

$G_{f}$ is the tangle described in Corollary 2 (i.e., the numerator $N\left(G_{f}\right)$ of $G_{f}$ is the unlink and its denominator $D\left(G_{f}\right)$ is the Terasaka knot $\left.K_{f}\right)$. 


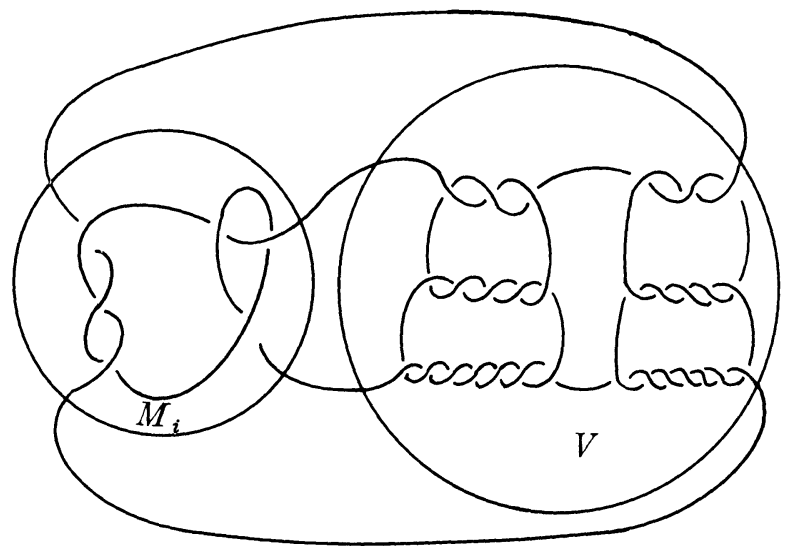

FIGURE 11

$C_{p_{l}}$ is a prime algebraic tangle such that its numerator $N\left(C_{p_{l}}\right)$ is the knot $K_{p_{0}, \ldots, p_{l}}$ described in Theorem 5 (such a prime tangle can be taken, for instance, as the one described in the proof of Proposition 4).

The knot $L$, join of prime tangles $G_{f}$ and $C_{p_{l}}$ is, therefore, by construction, prime and concordant to the prime knot $K_{p_{0}, \ldots, p_{l}}$.

The Conway polynomial of the knot $L$ is:

$$
\nabla(L)=\nabla\left(N_{G f}\right) \nabla\left(D_{C_{p_{l}}}\right)+\nabla\left(N_{C_{p_{l}}}\right) \nabla\left(D_{G j}\right)=\nabla\left(N_{C_{p_{l}}}\right) \nabla\left(D_{G j}\right) .
$$

(As $N_{G_{f}}$ is an unlink, $\nabla\left(N_{G_{f}}\right)=0$.) We have therefore, with more simplification, a result of Bleiler [1]

Corollary 5. Given polynomials $f(t)$ and $g(t)$ in $\mathbf{Z}\left[t, t^{-1}\right]$ with $g(t)$ $=g\left(t^{-1}\right)$ and $f(1)=g(1)=1$, there exist prime concordant knots $K, L$ with their Alexander polynomial $A_{K}(t)=g(t)$ and

$$
A_{L}(t)= \pm t^{m} g(t) f(t) f\left(t^{-1}\right) .
$$

Acknowledgements. I would like to thank the Department of Mathematics of the University of Texas for its kind hospitality. I am grateful to the referee for his careful reading of the first version of the manuscript and his suggestions for improvements.

\section{REFERENCES}

1. S. A. Bleiler, Realizing concordant polynomials with prime knots, Pacific J. Math. (to appear).

2. F. Bonahon and L. Siebenmann, (to appear).

3. J. H. Conway, An enumeration of knots and links, Computational Problems in Abstract Algebra (Pergamon Press, Oxford and New York, 1969), 329-358.

4. R. H. Fox and J. Milnor, Singularities of 2-spheres in 4-space and cobordism of knots, Osaka J. Math. 3 (1966), 257-267. 
5. C. McA. Gordon, Problems in knot theory, Lecture Notes in Math. 685 (SpringerVerlag, Berlin and New York, 1978), 309-311.

6. L. Kauffman, The Conway polynomial, Topology 20 (1981), 101-108.

7. R. C. Kirby and W. B. R. Lickorish, Prime knots and concordance, Math. Proc. Camb. Phil. Soc. 86 (1979), 437-441.

8. W. B. R. Lickorish, Prime tangles and knots, Transactions of the American Math. Society 267 (1981), 321-332.

9. J. M. Montesinos, Surgery on links and double branched covers of $S^{3}, K n o t s$, groups and 3-manifolds, Ann. of Math. Studies 84, 227-259.

10. K. Murasugi, On a certain subgroup of an alternating link, Amer. J. Math. 85 (1963), $544-550$.

11. D. Rolfsen, Knots and links, Publish and Perish (1976).

12. H. Schubert, Knoten mit 2 Brücken, Math. Z. 65, 133-170.

13. L. Siebenmann, Exercises sur les noeuds rationnels, polycopie, Orsay (1975).

14. H. Terasaka, On null-equivalent knots, Osaka Math. J. 11 (1959), 95-113.

15. F. Waldhausen, Eine Klasse von 3-đimensionalen Mannigfaltigkeiten II, Invent. Math. 4 (1967), 87-117.

Université de Genève,

Genève, Suisse 